\title{
Composition of 298 Baptistina: Implications for the K/T impactor link
}

\author{
Vishnu REDDY ${ }^{*}$, Joshua P. EMERY ${ }^{2}$, Michael J. GAFFEY ${ }^{1}$, William F. BOTTKE ${ }^{3}$, Abigail CRAMER ${ }^{4,5}$, \\ and Michael S. KELLEY ${ }^{4,6}$ \\ ${ }^{1}$ Department of Space Studies, University of North Dakota, Grand Forks, North Dakota 58202, USA \\ ${ }^{2}$ Department of Earth and Planetary Sciences, University of Tennessee, Knoxville, Tennessee 37996, USA \\ ${ }^{3}$ Southwest Research Institute, 1050, Walnut St., Suite 300, Boulder, Colorado 80302, USA \\ ${ }^{4}$ Department of Geology and Geography, Box 8149, Georgia Southern University, Statesboro, Georgia 30460, USA \\ ${ }^{5}$ Present address: S\&ME, Inc., 7604 Waters Ave., Savannah, Georgia 31406, USA \\ ${ }^{6}$ Present address: Planetary Science Division, Science Mission Directorate, NASA Headquarters, Washington, D.C. 20546, USA \\ ${ }^{*}$ Corresponding author. E-mail: vishnu.kanupuru@und.nodak.edu
}

(Received 08 December 2008; revision accepted 14 June 2009)

\begin{abstract}
Bottke et al. (2007) suggested that the breakup of the Baptistina asteroid family (BAF) $160^{+30}{ }_{-20}$ Myr ago produced an "asteroid shower" that increased by a factor of $2-3$ the impact flux of kilometer-sized and larger asteroids striking the Earth over the last $\sim 120 \mathrm{Myr}$. This result led them to propose that the impactor that produced the Cretaceous/Tertiary (K/T) mass extinction event $65 \mathrm{Myr}$ ago also may have come from the BAF. This putative link was based both on collisional/dynamical modeling work and on physical evidence. For the latter, the available broadband color and spectroscopic data on BAF members indicate many are likely to be dark, low albedo asteroids. This is consistent with the carbonaceous chondrite-like nature of a 65 Myr old fossil meteorite (Kyte 1998) and with chromium from $\mathrm{K} / \mathrm{T}$ boundary sediments with an isotopic signature similar to that from CM2 carbonaceous chondrites. To test elements of this scenario, we obtained near-IR and thermal IR spectroscopic data of asteroid 298 Baptistina using the NASA IRTF in order to determine surface mineralogy and estimate its albedo. We found that the asteroid has moderately strong absorption features due to the presence of olivine and pyroxene, and a moderately high albedo ( $20 \%)$. These combined properties strongly suggest that the asteroid is more like an S-type rather than Xc-type (Mothé-Diniz et al. 2005). This weakens the case for 298 Baptistina being a CM2 carbonaceous chondrite and its link to the K/T impactor. We also observed several bright (V Mag. $\leq 16.8$ ) BAF members to determine their composition.
\end{abstract}

\section{INTRODUCTION}

Catastrophic events have strongly affected the course of evolution of life on planet Earth. Bottke et al. (2007) argued that a factor of 2 increase in the terrestrial and lunar impact flux over the last $\sim 120 \mathrm{Myr}$ (potentially including the K/T impactor) was likely triggered by the catastrophic disruption of a $\sim 170 \mathrm{~km}$ body in the inner region of the main asteroid belt. They identified the Baptistina asteroid family (BAF) as the probable source of this putative increase using a range of observational data and numerical modeling work (e.g., the orbital and absolute magnitude distribution of the observed family, asteroid color data from the Sloan Digital Sky Survey, and the existing spectroscopy of two large Baptistina family members, collisional and dynamical modeling work). According to Bottke et al. (2007), the BAF formed $160^{+30} /-20$ million years ago and since then the family members have been drifting both toward and away from the Sun via nongravitational Yarkovsky forces. This has allowed $10-20 \%$ of the kilometer-sized and larger BAF members, which once may have exceeded 140,000 members, to reach two nearby mean motion resonances (the 7:2 and 5:9 mean motion resonances with Jupiter and Mars, respectively) that in turn delivered them to orbits that cross those of the terrestrial planets. Numerical modeling work shows that asteroids exiting these resonances have a nearly $2 \%$ chance of striking the Earth. Taken together, Bottke et al. (2007) used these values to show that the BAF could have potentially dominated the background impact flux on the Earth, Moon, and other terrestrial planets for several tens of Myr, with the peak of the flux occurring about $100 \mathrm{Myr}$ ago.

Based on this premise, Bottke et al. (2007) speculated that there could be a connection between the putative asteroid shower produced by the BAF and the K/T impact, presumably 
the largest impact on Earth over the last 160 Myr. Using dynamical modeling work as well as information on the nature of the $\mathrm{BAF}$ and the $\mathrm{K} / \mathrm{T}$ impactor, they estimated there was a $\sim 90 \%$ probability that the $\mathrm{K} / \mathrm{T}$ impactor was derived from the BAF asteroid shower.

In order to perform the $\mathrm{K} / \mathrm{T}$ calculation, Bottke et al. (2007) checked to see whether the BAF's taxonomic properties were consistent with what was known about the $\mathrm{K} / \mathrm{T}$ impactor. The deduced composition of the $\mathrm{K} / \mathrm{T}$ impactor, while somewhat murky, is consistent with it coming from a dark, low albedo asteroid. An investigation of a $65 \mathrm{Myr}$ old highly-altered fossil meteorite, presumably a fragment from the $\mathrm{K} / \mathrm{T}$ impactor, was found to be most similar to carbonaceous chondrite meteorites, themselves a dark meteorite class (Kyte 1998). Similarly, chromium isotope studies of sediments from several different $\mathrm{K} / \mathrm{T}$ sites (Trinquier et al. 2006; see also Shukolyukov and Lugmair 1998) suggested the impactor was most similar to CM2-type carbonaceous chondrites. Assuming the latter is true and that the BAF indeed produced the $\mathrm{K} / \mathrm{T}$ impactor, it would suggest that the BAF was likely similar in composition to CM2-type carbonaceous chondrites.

Here we attempt to further test the BAF-K/T impactor link by observing and spectrally characterizing some of the brightest objects in the BAF. Our goals in this particular paper are to determine whether 298 Baptistina is spectrally consistent with CM2-type carbonaceous chondrites, and thereby test the $\mathrm{K} / \mathrm{T}$ impactor link. The compositional investigation of smaller BAF members, such as those identified by Parker et al. (2008) using SDSS colors, is ongoing and will be described in a follow-up paper. It is also important to recall what these taxonomic classifications mean and their relationship (or the lack of) to meteorites. Taxonomic classification is a useful technique to broadly classify large number of objects for applications such as identifying broad trends throughout the main belt or constraining membership of dynamical families, but it does not equate with compositional characterization of asteroids.

The largest member of the BAF, 298 Baptistina, has been classified as Xc-type based on its visible spectrum (Lazzaro et al. 2004). In this paper, we present new near-infrared ( 0.8 to $2.5 \mu \mathrm{m})$ spectra that we obtained in order to assess the surface composition of Baptistina. This spectral range is more diagnostic of actual silicate mineralogy than the visible region, and will therefore provide a test of the hypothesized CM2-like composition for Baptistina. We also present a measurement of thermal emission from Baptistina and the resulting albedo estimate. Albedo provides a significant constraint on compositional interpretation, with a CM2 assemblage having an albedo in the 3-6\% range (Gaffey 1976) while an S-type object would normally have an albedo in the $\sim 10-35 \%$ range (Gaffey et al. 1993a). We also observed several bright (V Mag. s16.8) BAF members to determine their composition and if they make geologic sense as members of the family.

\section{OBSERVATION AND DATA REDUCTION}

In an effort to constrain the composition of 298 Baptistina, its albedo and diameter, and the composition of some of its family members, an observational campaign was launched in Spring 2008 using the SpeX instrument (Rayner et al. 2003) at the NASA IRTF. Baptistina was observed in low-resolution prism mode $(0.75-2.50 \mu \mathrm{m})$ on Feb. 28, 2008 (13:30-14:30 UT), March 21, 2008 (13:00-13:30 UT), and March 22, 2008 (13:00-14:00 UT) and in long crossdispersion mode (LXD) (1.90-4.20 $\mu \mathrm{m})$ on March 21, 2008 (11:20-11:45 UT) (Table 1). All observations were made on site during which local standard stars and solar analog stars were also observed for extinction and solar continuum correction of the asteroid spectrum.

The low resolution prism data $(0.75-2.50 \mu \mathrm{m})$ were processed using methods described in Abell (2003) and the LXD data $(1.90-4.20 \mu \mathrm{m})$ were processed using methods described in Emery and Brown (2003).

\section{ALBEDO AND DIAMETER ESTIMATION}

Albedo measurement of an asteroid is a valuable datum that can constrain its compositional characterization. In the case of 298 Baptistina it could help confirm the compositional link to a specific meteorite type. Albedo measurements of Baptistina were not available prior to this study. In an effort to constrain the albedo, cross dispersion data $(1.90-4.20 \mu \mathrm{m})$ (Fig. 1) were obtained using the SpeX instrument on NASA IRTF.

\section{Albedo Estimation}

The spectrum of Baptistina measured at the IRTF on 21 March 2008 rises steeply longward of about $3.6 \mu \mathrm{m}$ (Fig. 1). This is interpreted as a contribution to the spectrum by thermal radiation from the surface, and we use this thermal tail to estimate the albedo of Baptistina. This is part of the test to see whether Baptistina is consistent with a carbonaceous chondrite-like composition, which would imply a low (few percent) albedo.

The approach used for estimating the asteroid's albedo is similar to that used by several other authors in the interpretation of relative spectral data of near-Earth asteroids (e.g., Abell 2003; Rivkin et al. 2005; Reddy et al. 2007; DeMeo and Binzel 2008). The data are not photometric (i.e., no reliable absolute flux calibration), prohibiting the normal application of the radiometric method to determine both size and albedo. Nevertheless, the relative spectral accuracy is quite good, and the location and magnitude of the thermal contribution provides an indication of the surface temperature and, since the location of the asteroid is well known, the albedo.

The thermal excess at each wavelength, $\gamma_{\lambda}$ (Rivkin 
Table 1.

\begin{tabular}{|c|c|c|c|c|c|c|c|}
\hline Asteroid & $\begin{array}{l}\text { Rotation period } \\
\text { (h) }\end{array}$ & $\begin{array}{l}\text { Taxonomic } \\
\text { classification }\end{array}$ & $\begin{array}{l}\text { Observing dates } \\
\text { (UT) }\end{array}$ & $\begin{array}{l}\text { Visual } \\
\text { magnitude }\end{array}$ & Airmass & $\begin{array}{l}\text { Phase } \\
\text { angle }\end{array}$ & $\begin{array}{l}\text { Heliocentric distance } \\
\text { (AU) }\end{array}$ \\
\hline \multirow[t]{3}{*}{298 Baptistina } & \multirow[t]{3}{*}{16.22} & \multirow[t]{3}{*}{$\mathrm{Xc}$} & 28 -Feb-08 & 14.4 & 1.14 & 22.5 & 2.109 \\
\hline & & & 21-Mar-08 & 13.85 & 1.246 & \multirow[t]{2}{*}{13.9} & \multirow[t]{2}{*}{2.128} \\
\hline & & & 22-Mar-08 & 13.85 & 1.28 & & \\
\hline 1126 Otero & & A & 21-Mar-08 & 14.68 & 1.17 & 18.3 & 1.96 \\
\hline 1365 Henyey & & S & 23-Mar-08 & 14.15 & 1.18 & 8.9 & 2.095 \\
\hline \multirow[t]{2}{*}{1619 Ueta } & \multirow[t]{2}{*}{2.94} & \multirow[t]{2}{*}{ S } & 22-Mar-08 & 16.5 & 1.06 & 27.1 & 2.182 \\
\hline & & & 23-Mar-08 & 16.5 & 1.041 & 27.1 & 2.184 \\
\hline 2093 Genichesk & 11.02 & $\mathrm{C}$ & 23-Mar-08 & 16.39 & 1.04 & 16.9 & 2.392 \\
\hline \multirow[t]{2}{*}{4375 Kiyomori } & & \multirow[t]{2}{*}{ A } & 22-Mar-08 & 14.92 & 1.13 & 5.4 & 2.083 \\
\hline & & & 23-Mar-08 & 14.92 & 1.08 & 5.9 & 2.082 \\
\hline 4859 Fraknoi & & $\mathrm{S}$ & 23-Mar-08 & 16.82 & 1.08 & 26.1 & 2.106 \\
\hline
\end{tabular}

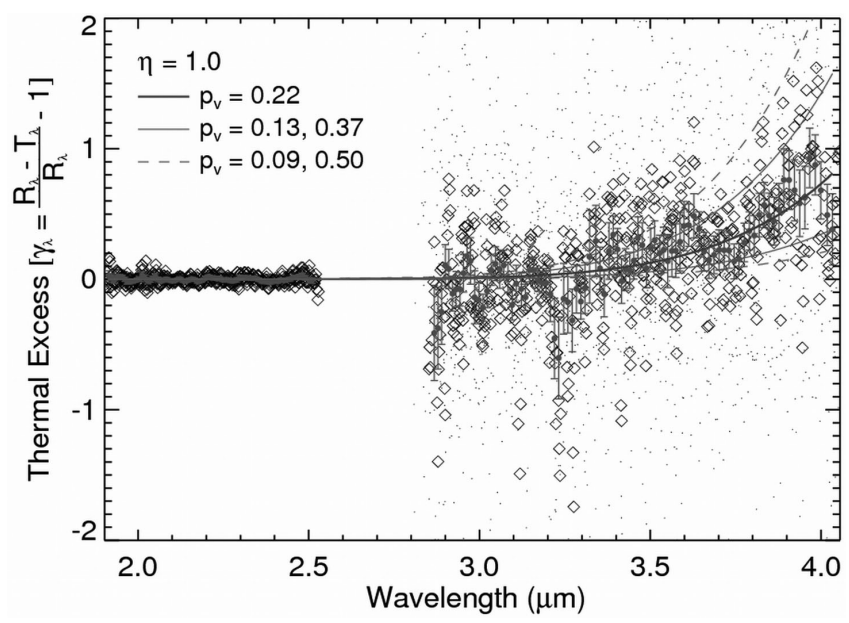

Fig. 1. Thermal IR data of 298 Baptistina obtained in the crossdispersion mode (LXD) using the SpeX instrument on NASA IRTF. The thermal models were calculated using NEATM. The black open diamonds are the data binned by 6 channels (which is the width of the slit), and the grey open circles are data binned by 36 channels.

et al. 2005) is calculated as

$$
\gamma_{\lambda}=\frac{\mathrm{R}_{\lambda}+\mathrm{T}_{\lambda}}{\mathrm{R}_{\lambda}}-1
$$

In this relation, $\mathrm{R}_{\lambda}$ represents the reflected component at a given wavelength, and $T_{\lambda}$ the thermal component. The quantity $R_{\lambda}+T_{\lambda}$ is the measured relative spectrum. Since we do not have an independent measure of $R_{\lambda}$ in the thermal region $(\lambda>$ $3.5 \mu \mathrm{m})$, the model is fit by a straight line to the K-band portion of the spectrum and extrapolating this line through the L-band. This straight line extrapolation is supported by the composition inferred from the prism (0.8 to $2.5 \mu \mathrm{m})$ spectrum (Fig. 2). Note that since both $R_{\lambda}$ and $T_{\lambda}$ are proportional to the square of the radius (i.e., the cross-sectional area), this factor cancels out and the thermal excess is independent of size. In other words, the solution for the albedo determined from $\gamma_{\lambda}$ is not degraded by our ignorance of the size of Baptistina.

The task is now to fit the thermal excess using modeled reflected and thermal fluxes. The modeled reflected flux is given by

$$
\mathrm{R}_{\lambda}^{\mathrm{m}}=\frac{\mathrm{F}_{\mathrm{sun}, \lambda}}{\mathrm{r}_{\mathrm{AU}}^{2}} \frac{\rho^{2}}{\Delta_{\mathrm{km}}^{2}} \Phi \mathrm{p}_{\lambda}
$$

where $\mathrm{r}_{\mathrm{AU}}$ is the heliocentric distance in $\mathrm{AU}, \Delta_{\mathrm{km}}$ is the geocentric distance in $\mathrm{km}$, and $\rho$ is the radius in $\mathrm{km} . \mathrm{F}_{\text {sun, }, \lambda}$ is the solar flux at $1 \mathrm{AU}$, for which the parameterization given in Smith and Gottlieb (1974) is used. The phase factor, $\Phi$, was calculated as described in Bowell et al. (1989) assuming a slope parameter, $\mathrm{G}$, of 0.15 , giving $\Phi=0.48$. Finally, $\mathrm{p}_{\lambda}$ is the geometric albedo at wavelength $\lambda$. This is related to the visible geometric albedo, $p_{v}$, by $p_{\lambda}=p_{v} f_{k v} R_{\lambda}$, where $f_{k v}$ is the ratio between the visible and K-band geometric albedos and $R_{\lambda}$ is the relative reflectance modeled as a straight line fit to the $\mathrm{K}$-band data as described above. The term $\mathrm{f}_{\mathrm{kv}}$ is estimated from the measured prism spectrum to be 1.12 .

The modeled thermal flux, $\mathrm{T}_{\lambda}^{\mathrm{m}}$, is calculated using a slight modification of the standard thermal model (STM; Lebofsky et al. 1986) that is commonly referred to as the nearEarth asteroid thermal model (NEATM; Harris 1998). The modifications are that the beaming parameter $(\eta)$, which was introduced to adjust the surface temperature to account for surface roughness but can be used to account for thermal inertia as well, is allowed to float rather than being set at a predetermined value and the mid-infrared phase function is handled slightly differently. Since $\eta$ adjusts the temperature, the choice of values for this parameter also affects the resulting albedo estimate. In a reanalysis of IRAS asteroid 
(298) Baptistina

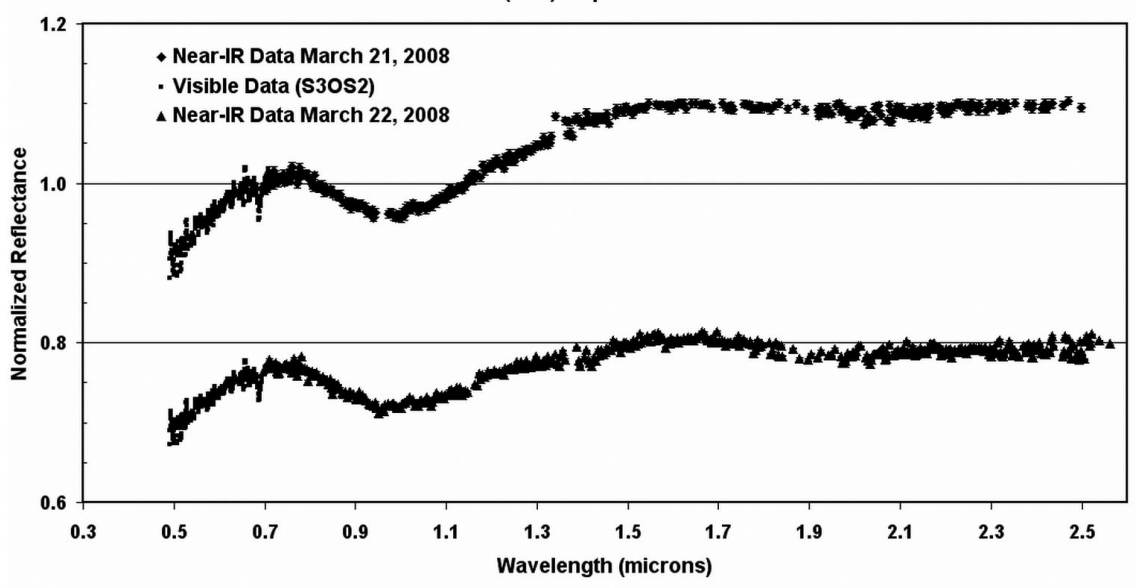

Fig. 2. Near-IR spectrum of 298 Baptistina obtained on March 21 (top) and 22 (bottom), 2008. The visible data from Lazzaro et al. (2004) and the near-IR data from NASA IRTF were combined to show the complete VNIR spectrum. Spectra from both nights are consistent with one another and are offset for clarity.

photometry, Russ Walker (personal communication) found that the distribution of $\eta$ in the Main Belt was a Gaussian with a mean near 1 and a standard deviation of $\sim 0.09$. In the thermal fits performed here, a default $\eta$ of 1.0 was adopted, but calculations were also performed with values of 0.9 and 1.1 to assess uncertainties in the albedo estimate.

The modeled thermal excess is compared to the measured thermal excess for a given choice of $p_{v}$ and $\eta$. The two are compared for goodness-of-fit by calculating a reduced $\chi^{2}$. A minimization algorithm (Press et al. 1992) was used to quickly and efficiently zero in on the best-fit $p_{v}$ for a given $\eta$. For $\eta=1$, the best-fit $p_{v}$ is 0.22 . The data are somewhat noisy and are reasonably well fit by a range of $\mathrm{p}_{\mathrm{v}}$ from 0.13 to 0.37 (Fig. 1). Taking account of the $1 \sigma$ range of $\eta$ mentioned above, the best-fit $p_{v}$ for $\eta=0.9$ is 0.29 and for $\eta=1.1$ is 0.17 . Adding together in quadrature the uncertainty due to the noise in the data and that from the $1 \sigma$ spread in $\eta$, the final albedo estimate is $\mathrm{p}_{\mathrm{v}}=0.22_{-0.10}^{+0.17}$ (i.e., range from 0.12 to 0.39 ). This derived albedo is much higher than the $\sim 3-$ $6 \%$ albedo expected for a carbonaceous chondrite-like body, but is in line with those of S-type asteroids, in agreement with the silicate absorption features apparent in the prism spectrum.

This albedo value is higher than the preliminary reported value of $14^{+2} /-3 \%$ by Reddy et al. (2008), which was based on data set that was not corrected for spectral slope. The error bars reported here are more realistic based on the scatter in the raw data.

\section{Diameter Estimation}

An asteroid's diameter can be calculated if its absolute magnitude $(H)$ and geometric visual albedo $\left(p_{v}\right)$ are known. $H$ is related to $p_{v}$ and the asteroid's effective diameter $\left(D_{e f f}\right)$ in kilometers by the equation (Fowler and Chillemi 1992):

$$
D_{e f f}=\frac{1329}{\sqrt{p_{v}}} * 10^{-\mathrm{H} / 5}
$$

Using an absolute magnitude $H=11.0$ and an albedo of $20^{+15} /-10 \%, 298$ Baptistina has a diameter of $\sim 19^{+7} /-5 \mathrm{~km}$. This diameter is much smaller than the $40 \mathrm{~km}$ reported by Bottke et al. (2007) which was estimated based on an assumed lower albedo.

\section{COMPOSITIONAL CHARACTERIZATION}

\section{Near-Infrared Spectra}

Asteroid composition is defined in terms of its mineralogy, mineral chemistry, and the relative abundances of major minerals. Key minerals (i.e., olivine, pyroxene) present on asteroid surfaces have diagnostic absorption features at visible to near-IR wavelengths $(0.30-2.50 \mu \mathrm{m})$ that can be used to determine surface compositions. These absorption bands arise from electronic transitions within a specific mineral structure. Several spectral band parameters (band center, band area ratios, band intensity) can be used to constrain an asteroid's mineralogy. The general position and pattern of the absorption band(s) are a function of the crystal structure, while the exact positions of the bands are a function of the mineral composition. The intensity of the feature is related to the abundance of the absorbing species (e.g., $\mathrm{Fe}^{2+}$ ) in the mineral and in a mineral mixture to the relative abundance of different minerals, the distortion of the mineral crystal structure, particle size, opaques, etc. Since a mineral is defined by its structure and composition, the positions and intensities of the absorption bands are uniquely related to specific minerals.

Figure 2 shows the average near-IR spectra of 298 Baptistina from March 21 and March 22, 2008 UT. Both 


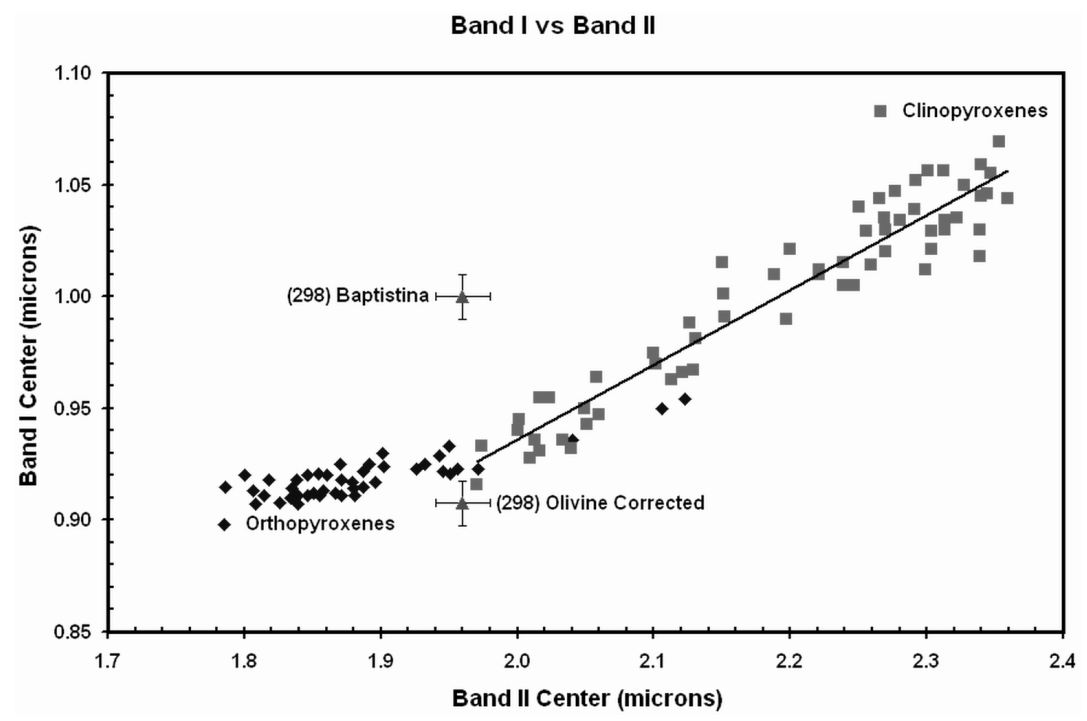

Fig. 3. Band-band plot showing the orthopyroxene to clinopyroxene trend line (Adams 1974). 298 Baptistina plots above the trend line indicating the presence of olivine.

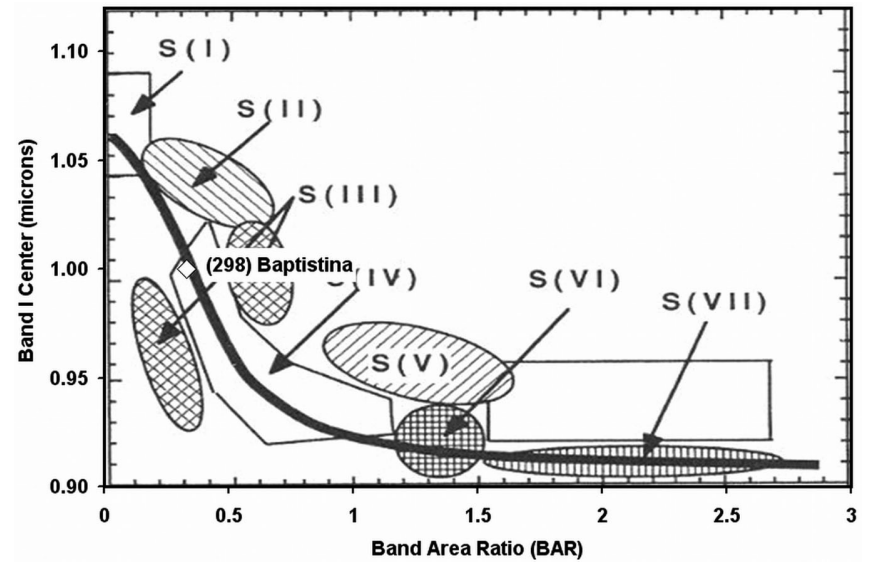

Fig. 4. S-asteroid subtypes plot from Gaffey et al. (1993b) showing the olivine-pyroxene mixing line. 298 Baptistina plots on this trend suggesting the presence of olivine and pyroxene in the surface assemblage.

spectra show a well resolved Band I absorption feature at $1.0 \pm 0.01 \mu \mathrm{m}$ with a band depth of $7 \pm 1 \%$ and a weaker Band II (band depth $2 \pm 1 \%$ ) feature at $1.96 \pm 0.02 \mu \mathrm{m}$. The Band II absorption feature is more apparent on March 22, 2008 data. The average Band Area Ratio (Band II Area/Band I Area) for both nights is $0.3 \pm 0.1$. Visible wavelength data $(\sim 0.50-0.75 \mu \mathrm{m})$ from Lazzaro et al. (2004) agree well with the near-IR data and confirm the earlier supposition that a weak feature may exist beyond $0.90 \mu \mathrm{m}$. The scatter at 1.4 and $1.9 \mu \mathrm{m}$ is due to incomplete correction of atmospheric water vapor features. Majaess et al. (2009) present a rotational lightcurve of Baptistina that was measured throughout March and April 2008 , finding a period of $16.23 \pm 0.02 \mathrm{~h}$. Since their observing period overlapped with some of our dates, we were able to match the near-IR spectral observations with rotational phase of the asteroid. Spectral observations on March 21, 2008, were made at $\sim 0.32\left(115^{\circ}\right)$ phase and $0.83\left(298^{\circ}\right)$ phase on March 22, 2008. As seen in Fig. 2 no significant rotational variation was observed in the measured spectral band parameters (band centers, band depth, Band Area Ratios [BAR]).

The presence of Band I and II strongly indicates the presence of a mafic silicate mineral-most likely pyroxene - on the asteroid's surface. Pyroxenes typically have Band I centers between 0.90-0.935 $\mu \mathrm{m}$ for low-Ca pyroxenes, 0.91-1.07 $\mu \mathrm{m}$ for Type B high-Ca pyroxenes and Band II centers between 1.78-1.97 $\mu \mathrm{m}$ for low-Ca pyroxenes and $\sim 1.97-2.36 \mu \mathrm{m}$ for Type B high-Ca pyroxenes (Cloutis and Gaffey 1991). For 298 Baptistina the Band I center of $1.0 \pm 0.01 \mu \mathrm{m}$ suggests that the surface assemblage has either a low- $\mathrm{Ca}$ and high-Ca pyroxene mixture or low- $\mathrm{Ca}$ and olivine mixture. Figure 3 shows a plot of Band I and II centers for ortho and clinopyroxenes. Baptistina plots above this trend line strongly suggesting the presence of olivine in the mixture.

Based on the Cloutis and Gaffey (1991) calibration the ratio of pyroxene in a mixture of olivine + pyroxene is $\sim 20: 80 \pm 10$. Correcting the Band I center for olivine using methods described in Gaffey et al. (2002), Baptistina plots below the trend line suggesting the presence of a possible third phase (high-Ca pyroxene) apart from olivine and low-Ca pyroxene. The Band I center and BAR of 298 Baptistina plot exactly on the olivine + orthopyroxene mixing trend line in the Gaffey S-asteroid subtype plots (Fig. 4) strengthening the case for an olivine and low-Ca pyroxene mixture. Based on the mineralogy, the ratio of olivine/pyroxene, and the location of the asteroid band parameters on S-asteroid subtype plot, LL chondrites are plausible meteorite analog for 298 Baptistina. 
Absorption features on 298 Baptistina are $7 \pm 1 \%$ (Band I) and $2 \pm 1 \%$ (Band II) deep, respectively. This is weaker than the average S-asteroid Band I depth (12.3 \pm 4.6\%) from Gaffey et al. (1993b), but within the range of S-asteroid Band I depth (2.7-24\%). The presence of weak/ suppressed features could be due to a variety of reasons. The presence of opaques (e.g., metal) suppresses silicate absorption features (Cloutis et al. 1990) and reddens the spectral slope (increasing reflectance with increasing wavelength). Cloutis (personal communication) noted reddening of spectral slope in olivine + metal mixtures with more than 50\% metal. Metal also tends to increase the albedo (moderate albedo $\sim 10-20 \%$ ). Shock-darkened ordinary chondrites also have lower albedo and subdued absorption features (Britt and Pieters 1994; Gaffey 1976). Other opaques (e.g., carbon) suppress spectral absorption features and decrease albedo (Clark 1981, 1983). A low abundance of the absorbing species (e.g., $\mathrm{Fe}^{2+}$ ) in a moderate albedo mineral can also cause weak features. Adams (1975) observed the increase in band depth and shifting of band center as $\mathrm{Fe}$ content of olivine increased in the forsterite-fayalite solid solution series. Presence of weakly featured minerals, for example low-Fe orthopyroxene can produce weak features with high albedos. Particle size and porosity can also affect the albedo and intensity of the absorption feature (Gaffey et al. 1993c). For transparent or weakly absorbing species, a decrease in particle size causes weakening of absorption features, but increases the albedo. For opaques such as carbon, decreasing particle size decreases albedo.

\section{CM2 Carbonaceous Chondrite Link}

Identifying a meteorite analog of an asteroid helps not only to link the meteorite to a source region in the asteroid belt but also to understand the petrologic history and formation conditions of the asteroid. Barring direct sampling and analysis of an asteroid, the most robust way to identify a meteorite analog of an asteroid to use spectral band parameters to determine the asteroid's surface mineralogy and mineral abundances.

\section{Visible Spectra}

The visible wavelength spectrum $(\sim 0.50-0.90 \mu \mathrm{m})$ of 298 Baptistina was published by Lazzaro et al. (2004) as part of the Small Solar System Object Spectroscopic Survey (S3OS2) and is shown in Fig. 5. The spectrum shows a rise in reflectance from $\sim 0.50-0.75 \mu \mathrm{m}$ and a drop in reflectance beyond that toward near-IR wavelengths suggesting a possible weak feature at $0.90 \mu \mathrm{m}$. The sharp narrow feature at $\sim 0.77 \mu \mathrm{m}$ is an uncorrected absorption due to atmospheric oxygen $\left(\mathrm{O}_{2}\right)$. Comparing the visible spectrum of 298 Baptistina with those of CM2 meteorites and meteorite mixtures (Fig. 5) shows a significant mismatch.

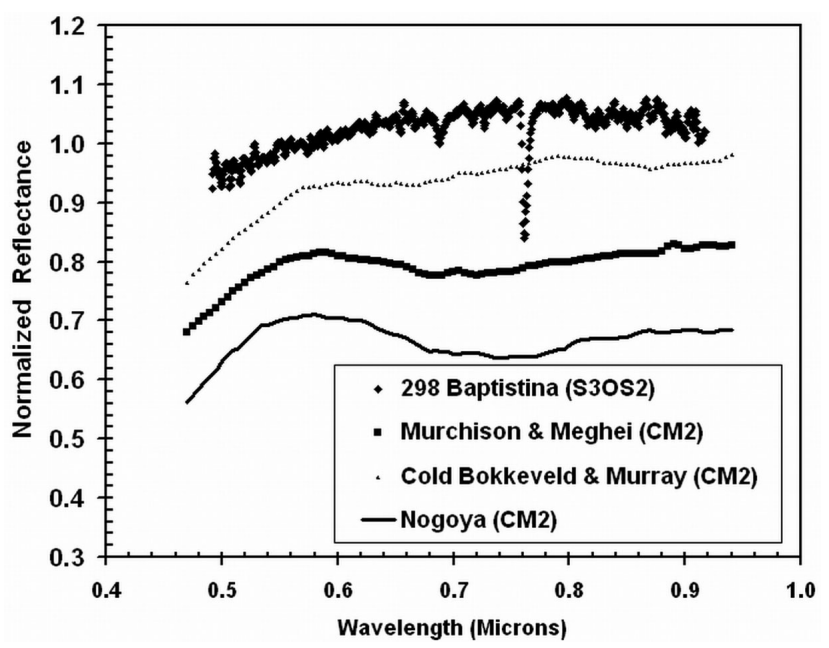

Fig. 5. Visible spectra of CM2 carbonaceous chondrites Cold Bokkeveld, Mighei, Murchison, Murray and Nogoya by Gaffey (1976) and 298 Baptistina from Lazzaro et al. (2004). Note the presence of broader feature centered near $\sim 0.70-\mu \mathrm{m}$ in Murchison, Mighei and Nogoya due to $\mathrm{Fe}^{2+}$-bearing in phyllosilicates which make up the bulk of the CM2 meteorites (e.g., Calvin and King (1997).

\section{Near-IR Spectra}

Apart from olivine and pyroxene there is also a possible third phase (probably an opaque phase such as metal or carbon) that is suppressing the absorption features. The albedo of the asteroid is very helpful in narrowing down possible meteorite analogs. Based on the preliminary albedo estimate using cross-dispersion data 298 Baptistina has a moderate albedo $\sim 20 \%$.

Using this information the first step is to investigate the link between 298 Baptistina and CM2 carbonaceous chondrites. The CM2 carbonaceous chondrites all belong to petrologic type 2; meaning they have undergone aqueous alteration but no thermal metamorphism (Brearley and Jones, 1998). Near-IR laboratory spectra of Cold Bokkeveld, Mighei and Nogoya show a weak absorption feature $(<8 \%)$ due to phyllosilicates at $\sim 0.90 \mu \mathrm{m}$, which is a complex feature with overlapping weaker bands (Gaffey 1976). No detectable Band II absorption feature is seen at $2.0 \mu \mathrm{m}$ and the spectral slope is redder (increasing reflectance with increasing wavelength) beyond $1.0 \mu \mathrm{m}$.

More recent spectral data of Antarctic CM2 meteorites from the RELAB database collected, by Pierre Hudson were analyzed for this study. Of the six samples, five were of type $\mathrm{CM} 2$ and one was CM1. Only two CM2 samples (Pecora Escarpment [PCA] 02012 and Queen Alexandra Range [QUE] 99038) have detectable Band I and II features but the band parameters (band centers, BAR, and band depth) of these CM2 carbonaceous chondrites and from Gaffey (1976) are inconsistent with 298 Baptistina (Fig. 6). Albedo measurements of $\mathrm{CM} 2$ carbonaceous chondrites by Gaffey 


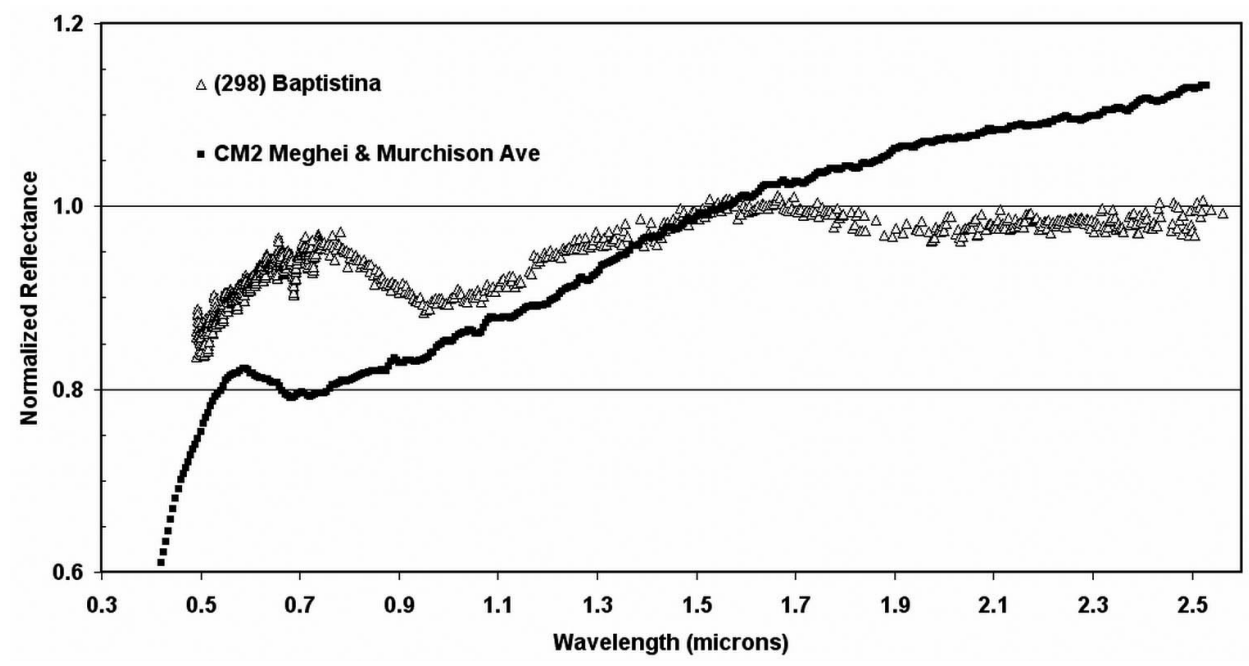

Fig. 6. Near-IR spectrum of 298 Baptistina and average spectrum of CM2 carbonaceous chondrite meteorites Mighei and Murchison (Gaffey 1976). The spectra have been normalized to unity at $1.50 \mu \mathrm{m}$.

(1976) shows most have albedos from $3.3 \%$ (Cold Bokkeveld) to $4.7 \%$ (Nogoya). Albedo measurements (absolute reflectance at $0.565 \mu \mathrm{m}$ ) of Antarctic CM2 samples show that all but two samples (PCA 02012 and QUE 99038) have low albedos between 2 and 5\%, similar to Gaffey (1976) measurements. PCA 02012 and QUE 99038 are minor to moderately weathered samples that have albedos of $\sim 13 \%$, which is considerably higher than all other known CM2 meteorite albedos. There is a strong possibility that terrestrial weathering has had some effect on the albedos of these meteorites. The spectral effects of terrestrial weathering on meteorites are not well characterized. Vilas and Gaffey (1989) suggested that such high albedos $(7-15 \%)$ are possible in highly aqueously altered CM2 assemblages, but the absorption features are still very weak $(<5 \%)$.

Many dark main belt asteroids have been associated with CM2 assemblages by previous researchers (Vilas and Gaffey 1989; Jones et al. 1990; Vilas and McFadden 1992; Vilas and Sykes 1996; Fornasier et al. 1999). Most of this research was done using visible spectra $(\sim 0.40-0.90 \mu \mathrm{m})$ and focused on identifying the $0.70 \mu \mathrm{m}$ feature due to charge transfer transition in phyllosilicates in CM2 meteorites. Most of the asteroids linked with CM2 carbonaceous chondrites have extremely low albedos (3.2-4.9\%) and maximum band depth of $<5 \%$ for the $0.70 \mu \mathrm{m}$ feature (Vilas and Gaffey 1989). Fornasier et al. (1999) observed 34 low-albedo ( 3-8\%) asteroids and found $65 \%$ have a weak feature (2-6\% deep) at $0.70 \mu \mathrm{m}$. In contrast 298 Baptistina has a more moderate albedo and no detectable feature at $0.70 \mu \mathrm{m}$.

Based on the absence of a $0.70 \mu \mathrm{m}$ feature, the presence of $1.0 \mu \mathrm{m}$ and $2.0 \mu \mathrm{m}$ features, quantified spectral band parameters (band centers, BAR, and band depth), and moderate albedos, it is reasonable to conclude that CM2 carbonaceous chondrites are not plausible meteorite analogs for 298 Baptistina.

\section{OTHER BAF MEMBERS}

To further investigate the Bottke et al. (2007) suggestion that smaller BAF members are dominated by dark C/X type objects we observed 13 other family members using the SpeX instrument on NASA IRTF. Table 1 lists6 of the 13 BAF members we have observed and their respective observational information. Of the 13 asteroids observed, we have completed data reduction and analysis for five. Here we present a brief summary of the compositional information for each of the BAF members based on their near-IR spectra.

\section{Otero}

With an estimated diameter of $\sim 11 \mathrm{~km}$ (based on $H=$ $12.1, p_{v}=0.25$ ), Otero is classified as an A-type under the Bus taxonomic classification (Bus and Binzel 2002) suggesting a nearly pure olivine phase as the major mineral. However, the near-IR spectrum of (1126) Otero (Fig. 7) shows a relatively deep band II (band depth $\sim 6 \%$ ) feature at $2.0 \mu \mathrm{m}$ indicating the presence of pyroxene along with olivine. Its band parameters are Band I center $0.98 \pm 0.01 \mu \mathrm{m}$, Band II center $2.0 \pm 0.01 \mu \mathrm{m}$ and BAR of 0.40 . Based on the calibration by Cloutis et al. (1986) the estimated abundance of pyroxene in a mixture of olivine and pyroxene is $25 \pm 5 \%$. Band parameters of (1126) Otero plot near the LL chondrite region of the bandband plot and in the S-IV region of the Gaffey S-asteroids subtype plot.

\section{Henyey}

This $\sim 12 \mathrm{~km}$ diameter asteroid (based on $H=11.7, \mathrm{pv}=$ 0.25 ) is a member of the Baptistina family and has been taxonomically classified as S-type by Lazzaro et al. (2004) as part of the Small Solar System Object Spectroscopic Survey 

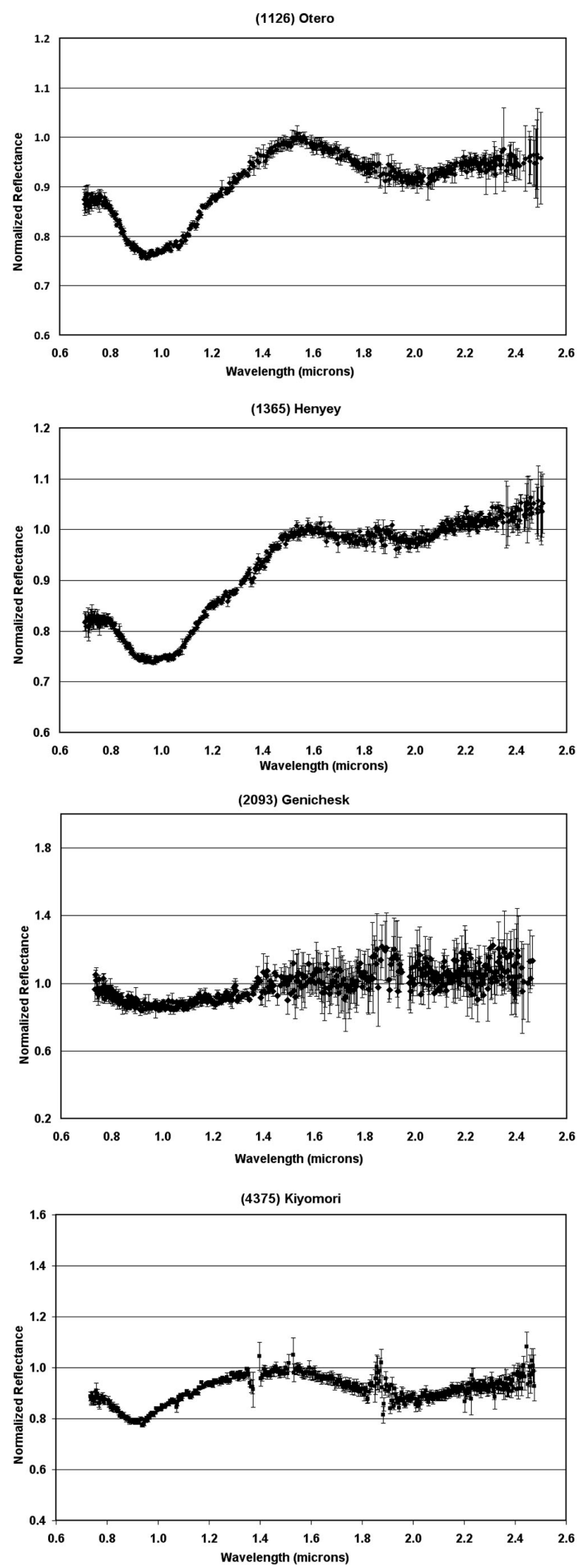

Fig. 7. Normalized near-IR reflectance spectrum of BAF members 1126 Otero, 1365 Henyey, 2093 Genichesk, and 4375 Kiyomori obtained using the SpeX instrument on NASA IRTF.
(S3OS2). Figure 7 shows the near-IR spectra of 1365 Henyey with deep absorption bands at 1- and $2-\mu \mathrm{m}$, and its band parameters are Band I center $1.01 \pm 0.01 \mu \mathrm{m}$, Band II center $2.01 \pm 0.01 \mu \mathrm{m}$ and BAR of 0.29 . The data plot above the low- and high-Ca mixing line on the band-band plot (Adams 1974) and above the S (IV) region of the Gaffey S-asteroids subtype plot. The estimated abundance of pyroxene in a mixture of olivine and pyroxene is $20 \pm 5 \%$ (Cloutis et al. 1986).

\section{Ueta}

A member of BAF that shows silicate absorption bands due to olivine and pyroxene. Due to low SNR of the spectra further analysis was not possible for this asteroid and more observations are planned during the next opposition.

\section{Genichesk}

An $\sim 18 \mathrm{~km}$ object (based on $H=12.6, \mathrm{pv}=0.05$ ) with an S3OS2 taxonomic classification of C-type. The near-IR spectrum of 2093 Genichesk (Fig. 7) shows a shallow, broad absorption feature at $\sim 1.0 \mu \mathrm{m}$ and a relatively flat spectrum beyond that. The shallow absorption feature is similar to $1.0 \mu \mathrm{m}$ absorption feature due to phyllosilicates on certain carbonaceous chondrites like Cold Bokkeveld (CM2) and CO3 meteorite Kainsaz from Gaffey (1976). Genichesk is the only object in the BAF we have observed and analyzed so far that has CM2 carbonaceous chondrites as possible meteorite analogs. Despite the possibility of Genichesk being a CM2 assemblage, its link to the BAF and ultimately the $\mathrm{K} / \mathrm{T}$ impactor is tenuous at best.

Note that while observing BAF members is straightforward, the identification of BAF members is not. The BAF is located in a dynamically complex region of the main belt where it is mixed with members of the Flora and Vesta families (Bendjoya and Zappala 2002; Bottke et al. 2007). This makes it difficult to sort out true BAF members from interlopers, particularly at the bright end of the absolute magnitude distribution where low albedo BAF members may be mixed with high albedo S- and V-class asteroids. The SDSS color data mainly exists for objects with $\mathrm{H} \sim 15-16$ and has limited coverage among brighter BAF members with $\mathrm{H}<14$. Moreover, until recently, the BAF was identified using cluster algorithms set to grab large numbers of nearby (and potentially unrelated) objects (Bottke et al. 2007). This may explain why the BAF was long thought to merely be a sub-cluster within the Flora family (Williams 1992), and why previous attempts to characterize bright BAF members have produced a potpourri of taxonomic types (Mothé-Diniz et al. 2005). These factors make it impossible to link Genichesk to the $\mathrm{BAF}$ or the $\mathrm{K} / \mathrm{T}$ impactor. 


\section{Kiyomori}

This asteroid has been classified as A-type by Lazzaro et al. (2004) as part of the Small Solar System Object Spectroscopic Survey (S3OS2). Figure 7 shows the near-IR spectrum of (4375) Kiyomori with moderately deep Band I and Band II features (13\% and $11 \%$ deep, respectively). The spectral band parameters of this asteroid are Band I center $0.93 \pm 0.01 \mu \mathrm{m}$, Band II center $2.0 \pm 0.01 \mu \mathrm{m}$ and BAR of 1.63. The data plot exactly on the low- and high-Ca mixing line on the band-band plot (Adams 1974) suggesting the presence of a nearly pure pyroxene assemblage. The asteroid falls in the basaltic achondrite region of the Gaffey Sasteroids subtype plot. The estimated abundance of olivine in a mixture of olivine and pyroxene is $<10 \pm 5 \%$ (Cloutis et al. 1986). Kiyomori is a classic example of the failure of taxonomic classifications in terms of providing compositional information. Based on the spectral band parameters and the inferred mineralogy (4375) Kiyomori is not an A-type (as suggested by Lazzaro et al. 2004).

\section{SUMMARY AND CONCLUSIONS}

Asteroid 298 Baptistina is a fascinating object with complex compositional characteristics and physical properties. Near-IR spectroscopic data for the asteroid show that its surface is dominated by a mineral assemblage of olivine and pyroxene. While the spectral features are subdued relative to the average S-type asteroid, the inferred mineralogy is inconsistent with CM2 carbonaceous chondrite meteorites. The estimate of a moderate albedo (despite large errors) also supports the argument that Baptistina has a composition similar to S-type asteroids. Our preliminary analysis suggests that possible meteorite analogs of Baptistina would include ordinary chondrites (possible LL chondrite), but we cannot exclude other meteorites like ureilites. However, it is important to note that identifying meteorite analogs for asteroids even using high SNR near-IR spectra is challenging at best due to the various observational and physical factors that affect spectral band parameters.

If 298 Baptistina is not a CM2 carbonaceous chondrite assemblage, what are the implications for the $\mathrm{K} / \mathrm{T}$ impactor link to the BAF? While one possibility is that the link is simply wrong, we suggest here that answer may not be straightforward. In fact, we believe there are several issues that need to be further explored.

First, one might consider the possibility that 298 Baptistina is atypical of BAF members. Recall that Parker et al. (2008) found the Baptistina family is distinct from the Flora and Vesta families in SDSS color data (as well as other S-type families across the main belt). According to their analysis, the average color of BAF members places them in their C-type category (with their other categories being S- and V-types). However, SDSS color classification schemes have little physical relationship to composition or mineralogy of an object. Given this ambiguity, it is impossible to confirm the compositional link between these C-type objects identified by Parker et al. (2008) and 298 Baptistina without detailed mineralogical characterization. More work on this topic is the subject of an upcoming paper by our group.

It is interesting to note that the $\sim 19^{+7}{ }_{/-5} \mathrm{~km}$ diameter estimated here for 298 Baptistina is very close to the one predicted by the size frequency distribution for the largest remnant of the breakup of the BAF by Bottke et al. (2007). This may indicate that there is a link between the unusual nature of 298 Baptistina and the BAF members found by Parker et al. (2008). It would require, however, that BAF members have very different albedos than 298. Thermal infrared measurements by Lazzaro et al. (personal communication) should further constrain the albedo and diameter of Baptistina. Based on the preliminary spectral analysis of other BAF members it is evident that they are compositionally diverse and not consistent with a CM2-type assemblage.

Finally, while the proposed CM2-chondrite link to 298 Baptistina is essentially disproved, it is important to point out that the compositional information we have on the $\mathrm{K} / \mathrm{T}$ impactor is not conclusive. For example, it is possible that the highly-altered fossil meteorite described by Kyte (1998) is indeed consistent with BAF member spectra. To date, there are no published investigations of this possibility. For such work to succeed, though, BAF material and presumably the fossil meteorite would need to have $\mathrm{Cr}$ isotope signatures similar to material found at the $\mathrm{K} / \mathrm{T}$ boundary, which happen to be similar to CM2-type carbonaceous chondrites.

Acknowledgments-We wish to express our gratitude for the stimulating reviews of the original manuscript provided by Tim McCoy and Dan Britt. Portions of this research (V. R., M. J. G.) were supported by NASA NEOO Program Grant NNX07AL29G and NASA Planetary Geology and Geophysics Grant NNX07AP73G [V. R., M. J. G.]. A. C. was supported by NASA Planetary Astronomy Grant NNG05GF90G (P. M. Asher, PI). The research utilizes spectra acquired by C. M. Pieters, and P. Hudson with the NASA RELAB facility at Brown University. V. R. would like to thank Dr. Paul Abell, Dr. Paul Hardersen, M. Schaal, D. Majaess, and D. Takir for their support in this research. We thank the IRTF TAC for awarding time to this project, and to the IRTF TOs and MKSS staff for their support.

\section{Editorial Handling-Dr. Andy Rivkin}

\section{REFERENCES}

Abell P. A. 2003. Near-IR reflectance spectroscopy of main belt and near-Earth objects: A study of their composition, meteorite affinities and source regions. Ph.D. thesis, Rensselaer Polytechnic Institute, Troy, New York, USA. 
Adams J. B. 1974. Visible and near-infrared diffuse reflectance spectra of pyroxenes as applied to remote sensing of solid objects in solar system. Journal of Geophysical Research 79:4829-4836.

Adams J. B. 1975. Interpretation of visible and near-infrared diffuse reflectance spectra of pyroxenes and other rock-forming minerals. In Infrared and Raman Spectroscopy of Lunar and Terrestrial Minerals, edited by Karr C., New York: Academic Press. pp. 91-116.

Bendjoya P. and Zappala V. 2002. Asteroid family identification. In Asteroids III, edited by Bottke W. F., Cellino A., Paolicchi P., and Binzel R. P. Tucson: The University of Arizona Press. pp. 613618.

Bottke W. F., Vokrouhlický D., and Nesvorný D. 2007. An asteroid breakup $160 \mathrm{Myr}$ ago as the probable source of the $\mathrm{K} / \mathrm{T}$ impactor. Nature 449:48-53.

Bowell E., Hapke B., Domingue D., Lumme K., Peltoniemi J., and Harris A. W. 1989. Application of photometric models to asteroids. In Asteroids II, edited by Binzel R. P., Gehrels T., Matthews M. S. Tucson: The University of Arizona Press. pp. 524-556.

Brearley A. J. and Jones R. H. 1998. In Planetary materials, edited by Papike J. J. Washington, D.C.: Mineralogical Society of America. pp. 1-370.

Britt D. T. and Pieters C. M. 1994. Darkening in black and gas-rich ordinary chondrite meteorites The spectral effects of opaque morphology and distribution. Geochimica et Cosmochimica Acta 58:3905-3919.

Bus S. J. and Binzel R. P. 2002. Phase II of the Small Main-Belt Asteroid Spectrographic Survey: A feature-based taxonomy. Icarus 158:106-145.

Calvin W. M. and King T. V. V. 1997. Spectral characteristics of ironbearing phyllosilicates: Comparison to Orgueil (CI1), Murchison and Murray (CM2). Meteoritics \& Planetary Science 32:693701.

Chapman C. R., Morrison D., and Zellner B. 1975. Surface properties of asteroids: A synthesis of polarimetry, radiometry, and spectrophotometry. Icarus 25:104-130.

Clark R. N. 1981. The spectral reflectance of water-mineral mixtures at low temperatures. Journal of Geophysical Research 86:30743086.

Clark R. N. 1983. Spectral properties of mixtures of montmorillonite and dark carbon grains: Implications for remote sensing minerals containing chemically and physically adsorbed water. Journal of Geophysical Research 88:10,635-10,644.

Cloutis E. A., Gaffey M. J., Jackowski T. L., and Reed K. L. 1986. Calibrations of phase abundance, composition and particle size distribution for olivine-orthopyroxene mixtures from reflectance spectra. Journal of Geophysical Research 91:11641-11653.

Cloutis E. A., Gaffey M. J., Smith D. G. W., and Lambert R. J. 1990. Metal-silicate mixtures: Spectral properties and applications to asteroid taxonomy. Journal of Geophysical Research 95:83238338.

Cloutis E. A. and Gaffey M. J. 1991. Pyroxene spectroscopy revisited: Spectral-compositional correlations and relationship to geothermometry. Journal of Geophysical Research 96:2280922826.

DeMeo F. and Binzel R. P. 2008. Comets in the near-Earth population. Icarus 194:436-449.

Emery J. P. and Brown R. H. 2003. Constraints on the surface composition of Trojan asteroids from near-infrared $(0.8-4.0 \mu \mathrm{m})$ spectroscopy. Icarus 164:104-121.

Fornasier S., Lazzarin M., Barbieri C., and Barucci M. A. 1999. Spectroscopic comparison of aqueous altered asteroids with CM2 carbonaceous chondrites meteorites. Astronomy and Astrophysics (Supplement) 135:65-73.
Fowler J. W. and Chillemi J. R. 1992. IRAS Asteroid Data Processing: The IRAS Minor Planet Survey. Philips Laboratory Technical Report PL-TR-92-2049. Jet Propulsion Laboratory, Pasadena, California. pp. 17-43.

Gaffey M. J. 1976. Spectral reflectance characteristics of the meteorite classes. Journal of Geophysical Research 81:905-920.

Gaffey M. J., Burbine T. H., and Binzel R. P. 1993a. Asteroid spectroscopy: Progress and perspectives. Meteoritics \& Planetary Science 28:161-187.

Gaffey M. J., Bell J. F., Brown R. H., Burbine T. H., Piatek J., Reed K. L., and Chaky D. A. 1993b. Mineralogic variations within the S-type asteroid class. Icarus 106:573-602.

Gaffey S. J., McFadden L. A., Nash D., and Pieters C. M. 1993c. Ultraviolet, visible and near-infrared reflectance spectroscopy: Laboratory spectra of geologic materials. In Remote geochemical analysis: Elemental and mineralogical composition, edited by Pieters C. M. and Englert P. A. J. New York: Cambridge University Press. pp. 43-77.

Gaffey M. J., Cloutis E. A., Kelley M. S., and Reed K. L. 2002. Mineralogy of Asteroids. In Asteroids III, edited by Bottke W. F., Cellino A., Paolicchi P., and Binzel R. P. Tucson: The University of Arizona Press. pp.183-204.

Harris A. W. 1998. A thermal model for near-Earth asteroids. Icarus 131:291-301.

Jones T. D., Lebofsky L. A., Lewis J. S., and Marley M. S. 1990. The composition and origin of the $\mathrm{C}, \mathrm{P}$, and $\mathrm{D}$ asteroids: Water as a tracer of thermal evolution in the outer belt. Icarus 88:172-192.

Kyte F. T. 1998. A meteorite from the Cretaceous/Tertiary boundary. Nature 396:237-239.

Lazarro D., Angeli C. A., Carvano J. M., Mothé-Diniz T., Duffard R., and Florczak M. 2004. S3OS2: The visible spectroscopic survey of 820 asteroids. Icarus 172:179-220.

Lebofsky L. A., Sykes M. V., Tedesco E. F., Veeder G. J., Matson D. L., Brown R. H., Gradie J. C., Feierberg M. A., and Rudy R. J. 1986. A refined 'standard' model for asteroids based on observations of 1 Ceres and 2 Pallas. Icarus 68:239-251.

Majaess D. J., Higgins D., Molnar L. A., Haegert M. J., Lane D. J., Turner D. G., and Nielsen I. 2009. New constraints on the asteroid 298 Baptistina, the alleged family member of the K/T impactor. Journal of the Royal Astronomical Society of Canada 3:7-10.

Mothé-Diniz T., Roig F., and Carvano J. M. 2005. Reanalysis of asteroid families structure through visible spectroscopy. Icarus 174:54-80.

Parker A., Ivezić Ž., Jurić M., Lupton R., Sekora M. D., and Kowalski A. 2008. The size distributions of asteroid families in the SDSS Moving Object Catalog 4. Icarus 198:138-155.

Press W. H., Flannery B. P., Teukolsky S. A., and Vetterling W. T. 1992. Numerical Recipes in C. In The art of scientific computing. New York: Cambridge University Press. pp. 394-455.

Rayner J. T., Toomey D. W., Onaka P. M., Denault A. J., Stahlberger W. E., Vaca W. D., Cushing M. C., and Wang S. 2003. SpeX: A medium-resolution 0.8-5.5 micron spectrograph and imager for the NASA Infrared Telescope Facility. Publication of the Astronomical Society of the Pacific 115:362-382.

Reddy V., Gaffey M. J., Abell P. A., and Hardersen P. S. 2007. Mineralogical investigation and thermal modeling of near-Earth asteroids (11405) 1999 CV3, 2000 BD19, 2003 SA224, and 2005 YY93. (abstract \#1338). 38th Lunar and Planetary Science Conference. CD-ROM.

Reddy V., Kelley M. S., Emery J. P., Gaffey M. J., Bottke W. F., Schaal M., Cramer A. P., and Takir D. 2008a. Composition of Baptistina asteroid family: Implications for K-T impactor link (abstract \#5257). Meteoritics \& Planetary Science 43:A130.

Rivkin A. S., Binzel R. P., and Bus S. J. 2005 Constraining near-Earth 
object albedos using near-infrared spectroscopy. Icarus 175:175180.

Shukolyukov A. and Lugmair G. W. 1998. Isotopic evidence for the Cretaceous-Tertiary impactor and its type. Science 282:927-930. Smith E. V. P. and Gottlieb D. M. 1974. Solar flux and its variations. Space Science Review 16:771-802.

Trinquier A., Birck J.-L., and Allégre J. C. 2006. The nature of the K/ $\mathrm{T}$ impactor. A ${ }^{54} \mathrm{Cr}$ reappraisal. Earth and Planetary Science Letters 241:780-788.

Vilas F. and Gaffey M. J. 1989. Identification of phyllosilicate absorption features in main-belt and outer-belt asteroid reflectance spectra. Science 246:790-792.

Vilas F. and McFadden L. A. 1992. CCD reflectance spectra of selected asteroids. I. Presentation and data analysis considerations. Icarus 100:85-94.

Vilas F. and Sykes M. 1996. Are low-albedo asteroids thermally metamorphosed? Icarus 124:483-489.

Williams J. G. 1992. Asteroid families-An initial search. Icarus 96: 251-280. 